\title{
Evaluation of the Performance and Hematocrit Independence of the HemaPEN as a Volumetric Dried Blood Spot Collection Device
}

\author{
Sigrid Deprez,,$^{\dagger}$ Lucía Paniagua-González, ${ }^{\ddagger \odot}$ Sofie Velghe, ${ }^{\dagger}$ and Christophe P. Stove* ${ }^{* \dagger \odot}$ \\ ${ }^{\dagger}$ Laboratory of Toxicology, Department of Bioanalysis, Faculty of Pharmaceutical Sciences, Ghent University, Ottergemsesteenweg \\ 460, 9000 Ghent, Belgium \\ ${ }^{\ddagger}$ Toxicology Service, Institute of Forensic Sciences, Faculty of Medicine, University of Santiago de Compostela, Rúa San Francisco \\ s/n, 15782 Santiago de Compostela, Spain
}

\section{Supporting Information}

\begin{abstract}
Dried blood spots (DBS) are often used as a less invasive alternative to venous blood sampling. Despite its numerous advantages, the use of conventional DBS suffers from the hematocrit (hct) effect when analyzing a subpunch. This effect could be avoided by using hct-independent sampling devices, of which the hemaPEN is a recent example. This device collects the blood via four integrated $2.74 \mu \mathrm{L}$ microcapillaries, each depositing the blood on a prepunched paper disc. In this study, we evaluated the technical performance of the hemaPEN devices, using an extensive bioanalytical validation and application on authentic patient samples. An LC-MS/MS method quantifying caffeine and its metabolite paraxanthine in dried whole blood (using the hemaPEN device) was fully validated, meeting all preset acceptance criteria. A comparative analysis of 91

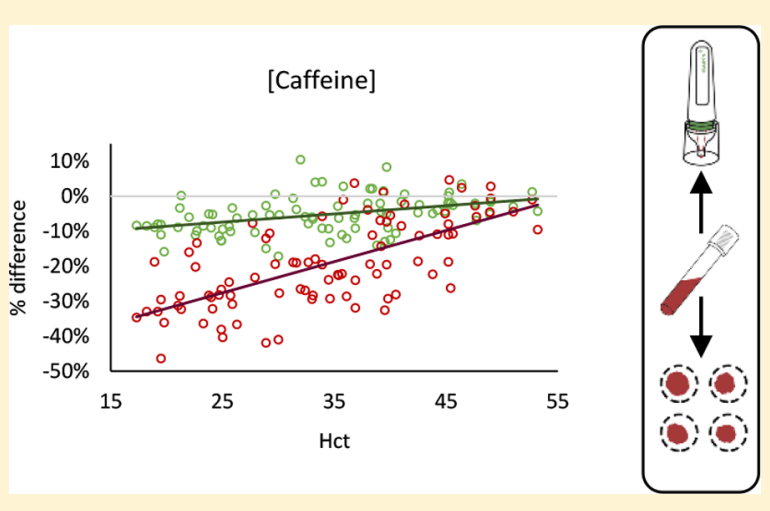
authentic patient samples (hct range: $0.17-0.53$ ) of hemaPEN, $3 \mathrm{~mm}$ DBS subpunches, and whole blood revealed a limited hct dependence ( $\leq 7 \%$ concentration difference over a $0.20-0.50$ hct range) for the hemaPEN devices, which we could not attribute to the analytical procedure. Using conventional partial-punch DBS ( $3 \mathrm{~mm}$ punches), concentration differences of $\geq 25 \%$ over this hct range were found. The hemaPEN showed to be robust to the effects of blood sample volume, device lot, analytical operator, and storage stability. The technical performance of the hemaPEN when dealing with patients having a high hct and in cases where a large blood drop is present should be further investigated. Based on the successful validation and application on patient samples, we conclude that the hemaPEN device shows good potential for the volumetric collection of DBS.
\end{abstract}

$\mathrm{M}$ icrosampling has gained popularity over the past decade because of its numerous advantages compared to conventional venous blood sampling. Many dried blood spot (DBS)-based methods have been developed for a variety of applications, e.g., phenotyping, therapeutic drug monitoring, doping analysis, or toxicology. ${ }^{1-7} \mathrm{~A}$ major drawback for implementing DBS in routine analyses is the presence of a hematocrit (hct) bias. The origin of this hct-based bias is threefold. $^{8-10}$ First, because viscosity of the blood is positively correlated with the hct, high-hct blood tends to spread less on filter paper compared to low-hct blood. As a result, when applying the same volume onto filter paper, smaller diameter spots are generated from higher hct blood compared to low-hct blood. Consequently, when taking a fixed subpunch of high-hct DBS, the analyte concentration is typically overestimated because of the larger volume that is present. This problem is well-known when using cellulose-based filter paper. A second aspect of the hct effect is the fact that it may impact the extraction efficiency and hence the recovery of the analyte. ${ }^{8-11}$ Generally, a high-hct DBS will form a certain barrier for extraction, resulting in a lower recovery and underestimation of the concentration. Third, samples with a different hct can be considered as different matrices, possibly causing an hctdependent matrix effect. ${ }^{8,9}$ In the past years, several dried blood sampling techniques distinct from conventional DBS collection have been developed such as the Mitra volumetric absorptive microsampling device (Neoteryx, USA), ${ }^{12,13}$ the HemaXis device (DBS system SA, Switserland), ${ }^{14}$ the volumetric absorptive paper disc (VAPD), mini-disc (VAPDmini) ${ }^{15}$ and the Capitainer device (KTH, Stockholm, Sweden). ${ }^{16,17}$ As most of these allow volumetric collection of blood, analysis of the complete dried microsample overcomes the impact of spreading on DBS-based quantitation. ${ }^{8,10}$ The recovery and matrix effect can still be influenced, though. Optimizing the extraction procedure and chromatographic conditions are an absolute requirement and therefore need to be evaluated during method validation. ${ }^{8,10,18}$ A recent addition to the panel of alternative devices is the hemaPEN (Trajan,

Received: July 13, 2019

Accepted: October 22, 2019

Published: October 22, 2019 
Melbourne, Australia), which is based on the volumetric application of blood onto prepunched filter paper discs. This device contains four integrated $2.74 \mu \mathrm{L}$ microcapillaries, which are filled by capillary action when touching a drop of blood. In a next step, following clicking of the device back into its base, the capillaries are emptied, depositing the blood onto four prepunched discs (3.5 mm diameter, PE 226 filter paper) that are integrated into the device. A successful proof-of-concept was already reported by Neto et al., ${ }^{19}$ who evaluated the accuracy and precision using $3 \mu \mathrm{L}$ end-to-end glass capillaries in conjunction with prepunched DBS discs. Furthermore, these results were compared to conventional low-volume blood collection systems, i.e., a micropipette and a digitally controlled analytical syringe. ${ }^{19}$ Both the accuracy and precision of dispensing a fixed volume onto filter paper were the same, or even better, with the capillary method when compared to the standard methods. In this study, we report the first in-depth evaluation of the technical performance of hemaPEN devices. This was achieved by an extensive bioanalytical validation and by application on authentic patient samples, using caffeine and its metabolite paraxanthine as model analytes. These analytes were chosen as it is easy to collect caffeine/paraxanthine positive samples, within a wide hct range, without the need to administer a substance. In this way, the accuracy and precision of the hemaPEN could be determined. A comparative analysis of hemaPEN DBS, $3 \mathrm{~mm}$ DBS subpunches, and whole blood was conducted to evaluate the hct independence of the hemaPEN vs. standard DBS technology. This comparison encompassed the analysis of 91 authentic patient samples with a broad hct range (0.17-0.53). Additional experiments on a subset of patient samples were set up to evaluate the robustness of the hemaPEN to the effects of blood sample volume, device lot, analytical operator, and storage stability. Besides evaluation of the analytical performance, we also evaluated the success rate of the device (i.e., whether all four capillaries were filled and dispensed for each sample), based on the data obtained in the validation and patient study.

\section{EXPERIMENTAL SECTION}

Chemicals and Stock Solutions. Caffeine, paraxanthine, the internal standards (ISs) caffeine- ${ }^{13} \mathrm{C}_{3}$ and paraxanthine- ${ }^{13} \mathrm{C}_{4^{-}}{ }^{15} \mathrm{~N}_{3}$, and formic acid (FA) were purchased from Sigma-Aldrich (Diegem, Belgium). LC-MS grade methanol was obtained from Biosolve (Valkenswaard, The Nederlands). Ultrapure water was produced by a Millipore purification system (Merck Millipore, Overijse, Belgium). A $1 \mathrm{mg} / \mathrm{mL}$ stock solution was prepared for both analytes by dissolving 10 $\mathrm{mg}$ of the compound in $10 \mathrm{~mL}$ of ultrapure water. For both the calibrators and QCs, independent $1 \mathrm{mg} / \mathrm{mL}$ stock solutions were made for caffeine and paraxanthine, and aliquots were stored at $-20{ }^{\circ} \mathrm{C}$. Working solutions, needed to generate calibrators and QCs, were freshly made on the day of analysis. For the IS, a commercial $1 \mathrm{mg} / \mathrm{mL}$ solution of caffeine $-{ }^{13} \mathrm{C}_{3}$ in methanol and $2 \mathrm{mg}$ of paraxanthine- ${ }^{13} \mathrm{C}_{4^{-}}{ }^{15} \mathrm{~N}_{3}$ was purchased and diluted to $100 \mu \mathrm{g} / \mathrm{mL}$ in methanol. Aliquots were stored at $-20{ }^{\circ} \mathrm{C}$. This $100 \mu \mathrm{g} / \mathrm{mL}$ stock solution was further diluted on the day of analysis of DBS to generate a solution in 80:20 $\mathrm{MeOH}: \mathrm{H}_{2} \mathrm{O}$, containing $0.01 \% \mathrm{FA}$, with a concentration of 40 $\mathrm{ng} / \mathrm{mL}$ caffeine $-{ }^{13} \mathrm{C}_{3}$ and $20 \mathrm{ng} / \mathrm{mL}$ paraxanthine $-{ }^{13} \mathrm{C}_{4^{-}}{ }^{15} \mathrm{~N}_{3}$. An aqueous working solution containing $5 \mu \mathrm{g} / \mathrm{mL}$ caffeine- ${ }^{13} \mathrm{C}_{3}$ and $2.5 \mu \mathrm{g} / \mathrm{mL}$ paraxanthine- ${ }^{13} \mathrm{C}_{4}{ }^{-15} \mathrm{~N}_{3}$ was prepared for the analysis of whole blood, as described by De Kesel et al. $^{20}$
Sample Collection. The use of blood from healthy volunteers and of left-over blood samples from patients was approved by the ethics committee of Ghent University Hospital (EC2018/0519). Blank venous whole blood was donated by a healthy female, caffeine abstinent donor in EDTA tubes (BD Vacutainer, New Jersey, USA) to generate calibrators and QCs on the day of analysis. Venous whole blood patient samples (EDTA-anticoagulated) were collected at Ghent University Hospital and were selected across several hospital departments to cover a wide hct range. HemaPEN devices were kindly provided by Trajan (Melbourne, Australia). To generate hemaPEN DBS, $20 \mu \mathrm{L}$ of venous whole blood was pipetted onto the top of a $2 \mathrm{~mL}$ Eppendorf, mimicking a drop of blood on a fingertip. The hemaPEN was then used to sample $4 \times 2.74 \mu \mathrm{L}$ by touching the drop of blood with the device while holding it horizontally and gently turning it. This results in filling of the four $2.74 \mu \mathrm{L}$ capillaries that are integrated in the device by capillary action. In a next step, the hemaPEN is pressed into a base and flipped to allow transfer of the blood onto four prepunched discs that are also integrated in the device. After sample collection was completed, the device was packed again in its original aluminum foil bag and left at room temperature to dry for at least $2 \mathrm{~h}$. No desiccant was added during storage, as desiccant is already integrated into the back of the hemaPEN. In addition, regular DBS were prepared by pipetting $50 \mu \mathrm{L}$ of whole blood onto Whatman 903 filter paper (GE Healthcare, Dassel, Germany). After drying for minimally $2 \mathrm{~h}$ at ambient room temperature, a $3 \mathrm{~mm}$ subpunch was taken for further analysis, which corresponds to a blood volume of approximately $3.5 \mu \mathrm{L}$ (the exact volume contained within this disc depends on the hct). A Sysmex XE5000 analyzer (Sysmex Corp., Kobe, Japan), run in manual mode, was used to determine the hat value of the whole blood, whenever necessary.

Sample Preparation. For the analysis of venous whole blood samples, $50 \mu \mathrm{L}$ of the specimen was transferred into a 2 $\mathrm{mL}$ Eppendorf tube. Consequently, $10 \mu \mathrm{L}$ of an aqueous IS solution was added followed by the addition of $100 \mu \mathrm{L}$ of $\mathrm{MeOH} / 0.01 \%$ FA. Samples were subsequently shaken for 10 $\mathrm{min}$ at $1000 \mathrm{rpm}$ at $22{ }^{\circ} \mathrm{C}$ and centrifuged for $10 \mathrm{~min}$ at $10000 \mathrm{~g}$ at ambient temperature. In a last step, $40 \mu \mathrm{L}$ of the supernatant was transferred to a new $2 \mathrm{~mL}$ Eppendorf tube, to which $130 \mu \mathrm{L}$ of $\mathrm{H}_{2} \mathrm{O} / 0.01 \%$ FA was added, prior to the transfer of $140 \mu \mathrm{L}$ to a glass vial with a plastic insert. For the analysis of hemaPEN DBS, a DBS retrieved from a hemaPEN device was transferred to a $2 \mathrm{~mL}$ Eppendorf tube. The analytes were extracted by adding $50 \mu \mathrm{L}$ of an 80:20 MeOH: $\mathrm{H}_{2} \mathrm{O}$ extraction solvent containing $0.01 \% \mathrm{FA}$ and the IS. Subsequently, hemaPEN DBS were shaken for $10 \mathrm{~min}$ at $1000 \mathrm{rpm}$ at $60{ }^{\circ} \mathrm{C}$, followed by centrifugation for $10 \mathrm{~min}$ at $10000 \mathrm{~g}$ at ambient temperature. A $30 \mu \mathrm{L}$ aliquot of the supernatant was transferred into a new $2 \mathrm{~mL}$ Eppendorf tube, and $130 \mu \mathrm{L}$ of $\mathrm{H}_{2} \mathrm{O} / 0.01 \%$ FA was added. Before analysis, 140 $\mu \mathrm{L}$ was transferred into a glass vial with a plastic insert. For conventional DBS analysis, $3 \mathrm{~mm}$ DBS subpunches were extracted by adding $70 \mu \mathrm{L}$ of an $80: 20 \mathrm{MeOH}: \mathrm{H}_{2} \mathrm{O}$ extraction solvent containing $0.01 \% \mathrm{FA}$ and the IS, shaking for $10 \mathrm{~min}$ at $1000 \mathrm{rpm}$ at $22{ }^{\circ} \mathrm{C}$ (or $60{ }^{\circ} \mathrm{C}$, when indicated), and centrifugating for $10 \mathrm{~min}$ at $10000 \mathrm{~g}$ at ambient temperature. To $45 \mu \mathrm{L}$ of the supernatant, $195 \mu \mathrm{L}$ of $\mathrm{H}_{2} \mathrm{O} / 0.01 \%$ FA was added prior to the transfer of $200 \mu \mathrm{L}$ into a plastic insert in a glass vial. 
UHPLC-MS/MS Method. For all samples, $10 \mu \mathrm{L}$ of extract was injected onto a Waters Acquity UPLC system (Waters, Milford, MA, USA), coupled to a SCIEX API 4000 mass spectrometer (SCIEX, Framingham, MA, USA). Waters Acquity console software and SCIEX Analyst version 1.6.2 controlled the LC-MS/MS system. Mobile phases A and B consisted of $0.01 \% \mathrm{FA}$ in $\mathrm{H}_{2} \mathrm{O}$ and $\mathrm{MeOH}$, respectively. The mass spectrometric and chromatographic conditions used for analysis of the whole blood, hemaPEN, and DBS samples were identical to those described by De Kesel et al. ${ }^{20}$

Validation of the hemaPEN Method. The hemaPEN method validation was based on the European Medicines Agency (EMA) and U.S. Food and Drug Administration (FDA) guidelines on bioanalytical method validation, ${ }^{21,22}$ also taking into account DBS-specific parameters, as outlined in the guideline by Capiau et al. ${ }^{18}$ The following parameters were evaluated during method validation: calibration model, homoscedasticity, accuracy and precision, carry-over, selectivity, lower limit of quantification (LLOQ), stability, matrix effect, and recovery. The calibration curve, set up in blood with an hct of 0.41 , ranged from 0.1 to $10 \mu \mathrm{g} / \mathrm{mL}$ for caffeine and from 0.05 to $5 \mu \mathrm{g} / \mathrm{mL}$ for paraxanthine. The nominal concentrations of the calibrators were $0.10,0.15,0.20,0.50$, $1.0,2.0,5.0$, and $10 \mu \mathrm{g} / \mathrm{mL}$ for caffeine and $0.050,0.10,0.15$, $0.20,0.50,1.0,2.0$, and $5.0 \mu \mathrm{g} / \mathrm{mL}$ for paraxanthine. The lower limit of quantification (LLOQ) was set at the level of the lowest calibrator. The low, medium, and high QCs had concentrations of $0.24 / 0.12 \mu \mathrm{g} / \mathrm{mL}, 2 / 1 \mu \mathrm{g} / \mathrm{mL}$, and $8 / 4 \mu \mathrm{g} /$ $\mathrm{mL}$ for caffeine/paraxanthine, respectively. The most suitable regression model was determined by calculating the sum percentage relative error (\%RE) for both unweighted and weighted $\left(1 / x, 1 / x^{2}, 1 / \sqrt{ } x, 1 / y, 1 / y^{2}\right.$, and $\left.1 / \sqrt{ } y\right)$ linear and quadratic regression models. Statistical evaluation to substantiate this choice was done using an R-script developed by Desharnais et al. ${ }^{23}$ Using this script, an F-test was performed to evaluate heteroscedasticity. If weighting was needed, a variance test for weight selection was done to select the most appropriate weighting factor. Additionally, a partial F-test was needed for model order selection. To verify the prerequisite for this test, a normal distribution of the standardized residuals, a Cramer von Mises test was performed. Accuracy and precision were evaluated by the analysis of four different QC levels over 4 different days, analyzed on each day in duplicate. The acceptance criterion for the \%bias was $15 \%$, except for the LLOQ where it was $20 \%$. The \%coefficient of variation $(\mathrm{CV})$ limit for the repeatability and total precision was set at 15 and at $20 \%$ for the LLOQ. One-way ANOVA analysis was performed to calculate the imprecision, as described by Wille et al. ${ }^{24}$ Carry-over was assessed by the injection of two blank samples (not containing analyte or IS in the extraction solvent) after the highest calibrator and was considered acceptable if the signal was less than $20 \%$ of the LLOQ analyte area and less than $5 \%$ of the IS area. The analysis of seven blank donor samples was used to evaluate the selectivity. A signal less than $20 \%$ of the LLOQ analyte area and less than $5 \%$ of the IS area was required to confirm the selectivity of the method. Long-term stability ( 2 months) was assessed at room temperature at low and high concentration levels $(0.12$ and $0.06 \mu \mathrm{g} / \mathrm{mL}$ and 8 and $4 \mu \mathrm{g} / \mathrm{mL}$ for caffeine and paraxanthine, respectively). Short-term stability (4 days) was checked at an elevated temperature of $60{ }^{\circ} \mathrm{C}$ at two concentration levels (Low QC and High QC). The acceptance criterion was less than $15 \%$ deviation of the nominal value. Matrix effect and recovery were assessed as described by Matuszewski, implying the evaluation of three sets of samples: one set spiked before extraction, another spiked after extraction, and a last set in the absence of the matrix to be assessed. ${ }^{25}$ In addition to using blank matrices from seven individual donors (hct $=0.40$ to 0.44 ), one donor sample was adapted to generate blood at two additional hct levels (0.20 and 0.58). The matrix effect was assessed at two different concentration levels (Low QC and High QC). The matrix factor was then calculated for each donor and can be defined as the ratio of the analyte peak area in the presence of matrix to the peak area in the absence of that same matrix. Also, the IS-normalized matrix effect was calculated by including the IS areas in the presence and absence of the matrix in the matrix factor. The EMA guidelines on bioanalytical method validation state that the CV (\%) on the IS-normalized matrix factor, calculated from the seven lots of matrix and two adapted hct matrices, should not exceed $15 \%$. To evaluate the recovery and a possible impact of the hct on the recovery, two sets of blood samples $(n=6)$ were prepared at three different hct levels $(0.21,0.41,0.60)$. A first set, prior to hemaPEN sampling and extraction, was spiked with analyte at two different concentration levels (Low QC and High QC). The second set was used for the hemaPEN-assisted collection of blank DBS, which were extracted using IS-containing extraction solvent and spiked with the theoretical amount of analyte present in $2.74 \mu \mathrm{L}$ of blood. Recovery (expressed as a $\%$ ) was calculated by dividing the peak areas at each condition in the set spiked before extraction by those of the corresponding samples spiked after extraction. The recoveries for the low- and high-hct blood were expressed relative to the extraction recovery for 0.41 hct blood. The recovery was considered hct-independent when the relative IS-compensated recovery for both low- and high-hct DBS was within $\pm 15 \%$ of the recovery for DBS with a 0.41 hct.

Application. A comparative study was conducted to compare caffeine and paraxanthine concentrations between hemaPEN DBS, $3 \mathrm{~mm}$ subpunches from DBS collected on Whatman 903 filter paper, and liquid whole blood. Left-over EDTA whole blood samples from hospital patients, preselected to ensure an even distribution in a wide hct range, were analyzed in duplicate to select caffeine and paraxanthine double positive samples (above the LLOQ for both analytes; $n$ =96). Analysis was performed based on a previously published method. ${ }^{20}$ Only positive samples for which the two whole blood measurements did not differ by more than $20 \%$ were included in the data set (for caffeine seven samples and for paraxanthine four samples were excluded based on the whole blood measurements). As an additional quality check for all analyses of the patient samples, in all instances, the paraxanthine to caffeine ratio was determined and compared to this ratio in liquid whole blood. Irrespective of the matrix used for analysis, this ratio should remain the same for a given sample. A generalized ESD test revealed one outlier (a conventional DBS sample), leading to the exclusion of this patient sample. This yielded an eventual data set of 88 samples for caffeine and 91 samples for paraxanthine. All positive samples were used for the generation of DBS using the hemaPEN and by pipetting $50 \mu \mathrm{L}$ of blood onto Whatman 903 filter paper to prepare regular DBS. Likewise, calibrators and QCs were generated using the hemaPEN or by pipetting blood onto filter paper. The use of isolated $2.74 \mu \mathrm{L}$ capillaries (as present in the hemaPEN) to manually prepare calibrators and 
Table 1. Within Day and Total Precision and Accuracy $(n=4 \times 2)$ for QCs of Caffeine and Paraxanthine at Four Concentration Levels (LLOQ, low, mid, and high) in hemaPEN.

\begin{tabular}{|c|c|c|c|c|c|c|}
\hline \multirow[b]{2}{*}{ QC } & \multicolumn{2}{|c|}{ within day precision $(\% \mathrm{CV})(n=4 \times 2)$} & \multicolumn{2}{|c|}{ total precision $(\% \mathrm{CV})(n=4 \times 2)$} & \multicolumn{2}{|c|}{ accuracy (\%bias) $(n=4 \times 2)$} \\
\hline & caffeine & paraxanthine & caffeine & paraxanthine & caffeine & paraxanthine \\
\hline LLOQ & 7.59 & 5.50 & 8.96 & 10.20 & 3.48 & -0.82 \\
\hline Low QC & 7.64 & 3.49 & 8.14 & 8.51 & 0.36 & 3.54 \\
\hline Mid QC & 3.46 & 5.08 & 8.26 & 7.16 & -5.38 & -4.21 \\
\hline High QC & 3.53 & 3.11 & 6.19 & 3.28 & -0.61 & 1.47 \\
\hline
\end{tabular}

QCs was considered and tried but was not practical and therefore not considered further. Since four DBS are generated per hemaPEN, incurred sample reanalysis could be performed by analyzing a second and third spot of the same device for each patient sample. Both incurred sample reanalyses were compared to the original hemaPEN analysis for both analytes by calculating the \% difference as (incurred sample reanalysis - hemaPEN DBS original measurement)/(mean of both analyses). At least two-thirds of the results should be within $20 \%$. This \% deviation was plotted against sample number according to increasing analyte concentration. The effect of the hct on the determination of caffeine and paraxanthine using the hemaPEN was assessed by plotting the $\%$ difference with whole blood as a function of the hct. The \% difference was calculated as the (hemaPEN DBS concentration - whole blood concentration)/whole blood concentration. The same plot was made for the $3 \mathrm{~mm}$ DBS subpunch to confirm the hct effect that should be present when using a DBS subpunch. ${ }^{13,16}$ In another set of patient samples with either low or high hct, the caffeine and paraxanthine concentrations, determined after full-spot analysis of DBS that were generated by pipetting 2.74 $\mu \mathrm{L}$ onto $3 \mathrm{~mm}$ prepunched filter paper discs (analyzed in triplicate), were compared with those from corresponding whole blood (analyzed in duplicate). The \% difference was calculated as [full DBS concentration (mean of triplicates) whole blood concentration (mean of duplicates)]/mean whole blood concentration and was plotted against the hct. Linear regression analysis was performed to identify a possible trend of the \% difference (hemaPEN DBS vs. whole blood or pipetted DBS vs. whole blood) as a function of the hct. Subsequently, the concordance between whole blood concentrations and hemaPEN DBS concentrations was investigated by Bland-Altman plots where the difference of both measurements divided by the mean (expressed as a \%) was plotted against the mean of both measurements. This allows a conclusion to be made if there is an overall over- or underestimation of the concentration obtained from the hemaPEN DBS compared to that in whole blood. Medcalc statistical software, version 14.12.0, was used to create BlandAltman plots.

The hemaPEN robustness to the effects of blood sample volume, device lot, analytical operator, and storage stability was evaluated using a subset $(n=25)$ of the caffeine/paraxanthine positive patient samples, carefully selected to ensure a spread across the entire available hct range ( 0.17 to 0.57$)$. The impact of blood volume was assessed by depositing $50 \mu \mathrm{L}$ (instead of $20 \mu \mathrm{L}$ ) of blood onto an Eppendorf cap to generate DBS using the hemaPEN. These two volumes were chosen as the minimum volume required to easily fill the four capillaries, which will in practice be close to $20 \mu \mathrm{L}$, whereas a $50 \mu \mathrm{L}$ blood drop lying on top of a fingertip can be considered already quite large. Alternatively, hemaPEN devices of another lot or hemaPEN devices of the same lot but handled by another operator were used. Two-thirds of the results should not vary by more than $20 \%$ of the mean of both concentrations (i.e., mean of original hemaPEN measurement and altered parameter measurement). The $\%$ difference is calculated as (altered parameter hemaPEN concentration - original hemaPEN concentration)/mean of both concentrations. Stability and the absence of extractability issues when working with authentic samples was assessed by storing hemaPEN devices for 4 days at $60{ }^{\circ} \mathrm{C}$ after drying at room temperature. Here, the mean deviation between the result and original concentration should not differ more than $15 \%$. This was calculated as (concentration after 4 days at $60{ }^{\circ} \mathrm{C}$ - original concentration)/original concentration. For the evaluation of all parameters related to hemaPEN robustness, analyses were performed in singlicate.

Additionally, a small transport simulation study was performed. Fingerstick samples were collected from 10 volunteers, using 2 hemaPEN devices, to prepare 2 sets of samples. Each volunteer provided a written informed consent (cfr. EC2018/0519). The fingerstick was performed using a $\mathrm{BD}$ microtainer contact-activated safety lancet (BD, Franklin Lakes, USA). One set of hemaPEN devices was retained in the laboratory, and the other replicate was posted from a nearby post office to the laboratory. In this way, transport and exposure to "real" environmental conditions were simulated. Per hemaPEN, two replicate spots were analyzed in the same batch against a freshly extracted calibration curve. Two-thirds of the results of the laboratory and transported samples must not vary by more than $20 \%$.

As the hemaPEN contains four $2.74 \mu \mathrm{L}$ capillaries that should each transfer blood onto the prepunched paper discs in the cartridge inside the pen, the success rate of filling and emptying of all capillaries was another parameter that could be assessed. For all samples (calibrators, QCs, and patient samples), the hemaPEN ID, the het of the blood, the number of capillaries filled with blood during sampling, and the number of capillaries where an actual transfer of blood took place were recorded. This allowed insight into the parameters affecting the functionality of the hemaPEN device.

\section{RESULTS AND DISCUSSION}

Method Validation. Calibration data from the four validation days $(n=8)$ were plotted. Homoscedasticity was determined by performing an F-test. As the calibration data were heteroscedastic, weighting was required, with $1 / x^{2}$ weighting being chosen based on the lowest sum \%RE. Using $1 / x^{2}$ linear models, the back-calculated concentrations were within $15 \%$ (with a single exception) for all calibrators for both analytes, justifying the use of this model. Statistical evaluation, using an R-script developed by Desharnais et al., ${ }^{23}$ substantiated this choice. For both analytes, a bias smaller than $6 \%$ was found at all concentration levels, as shown in Table 1. Both analytes could be determined in hemaPEN DBS with a 
repeatability below $8 \%(\mathrm{CV} \%)$ and a total imprecision below $11 \%(\mathrm{CV} \%)$. Selectivity and carry-over were within the preset limits ( $<20 \%$ of the LLOQ area and $<5 \%$ of the IS area). Absolute recovery was, for both analytes at each hct level, higher than $86 \%$. IS-compensated recovery ranged between 98 and $122 \%$. CV's on the IS-compensated recovery were below $9 \%$ and therefore within the preset acceptance criterion of $15 \%$. The recovery was hct-independent (i.e., within $\pm 15 \%$ of the recovery for hemaPEN DBS with a $0.41 \mathrm{hct}$ ), as depicted in Figure 1, although for the caffeine low QC, a statistically

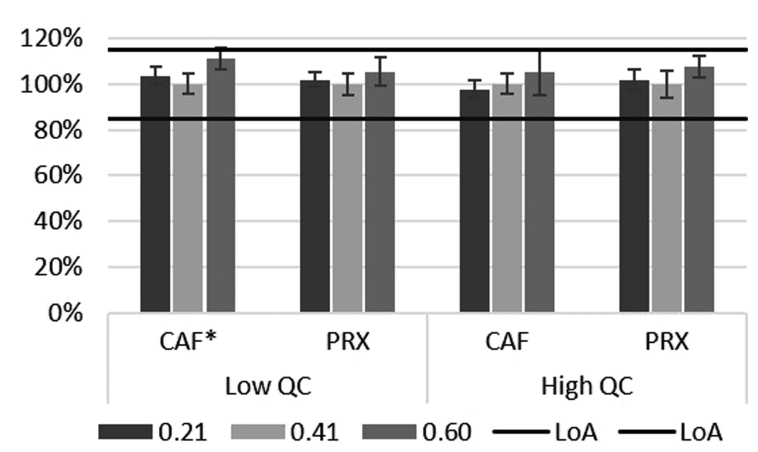

Figure 1. IS-compensated recovery (\%) of caffeine (CAF) and paraxanthine $(P R X)$ for hemaPEN DBS, measured at two concentration levels for different hct levels $(0.21 ; 0.41 ; 0.60)(n=$ 5 or 6) and relative to the recovery of a DBS with 0.41 hct. For the CAF Low QC spiked before extraction set, two outliers could be identified with a Dixon $Q$-test $(n=5$ for the calculation of the ISnormalized recovery factor for Low QC at het of 0.21 and 0.41 ). This was probably due to external contamination of the sample since only caffeine areas deviated. * indicates a statistically significant difference (two-sided ANOVA; $p<0.05$ ).

significant effect $(p<0.05)$ of the hct on the recovery was found. Post hoc analysis revealed a significant difference between the recovery for 0.60 hct DBS and both 0.41 and 0.21 hct DBS. Analyte matrix effects were for both analytes within 94-105\%, as shown in the Supplementary Data (Suppl. Data Table S-1). IS-compensated matrix effects were within 99$104 \%$, with CV's below 6\%, thus amply meeting the preset acceptance criterion of $15 \%$.

Both caffeine and paraxanthine were stable (i.e., within $11.5 \%$ of the nominal concentration) for at least 2 months at room temperature and 4 days at $60{ }^{\circ} \mathrm{C}$ when storing the hemaPEN devices in their original packages (Suppl. Data Table S-2). From these stability experiments, it can also be concluded that there are no "extractability" issues, when comparing very dry and freshly dried DBS. From all the abovementioned experiments, it can be concluded that the analytical validation of the hemaPEN, with inclusion of hct as an additionally evaluated parameter, was successful.

Application. Caffeine and paraxanthine were determined in duplicate (in independent runs) in venous whole blood patient samples (hct range $=0.17-0.53$; median $=0.345$ ), hemaPEN DBS, and $3 \mathrm{~mm}$ partial-punch DBS. For both analytes ( $n=88$ for caffeine and $n=91$ for paraxanthine), the mean of the duplicate whole blood measurements was defined as the reference concentration. Since four DBS spots are generated per hemaPEN, incurred sample reanalysis could be performed by analyzing a second and third spot of the same device for each patient sample. Both incurred sample reanalyses were compared to the original hemaPEN analysis for both analytes. As shown in Figure 2, for both caffeine and
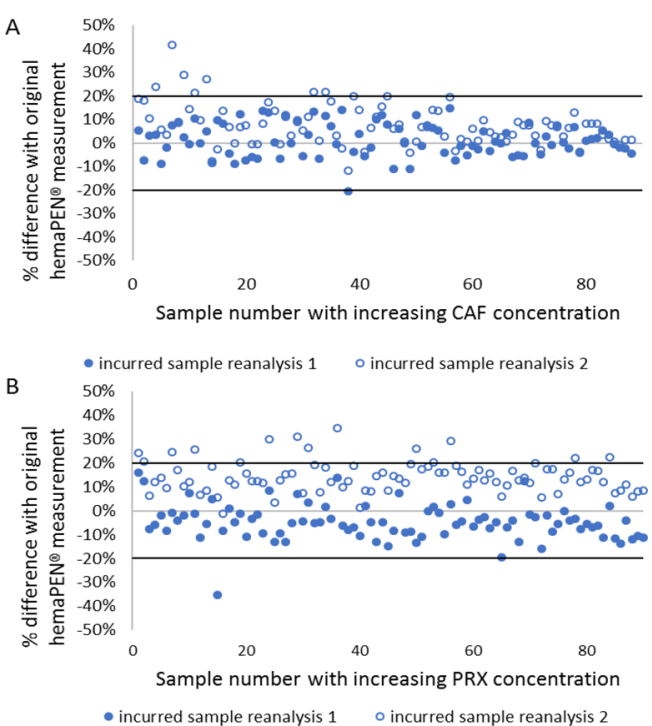

Figure 2. First and second incurred sample reanalyses compared to the original hemaPEN measurement for caffeine (A) and paraxanthine (B) in \% difference. The numbering of the $x$-axis refers to the number of samples, ranked here based on increasing concentration.

paraxanthine, the acceptance criterion of two-thirds of the concentrations having a difference of less than $20 \%$ was $^{\text {met. }}{ }^{21}$ For the first incurred reanalysis, $99 \%$ of the results for both analytes had a difference less than $20 \%$. For the second reanalysis, $92 \%$ of the caffeine concentrations and $85 \%$ of the paraxanthine concentrations differed less than $20 \%$ from the original hemaPEN measurement. An overall negative bias for the first reanalysis and positive bias for the second reanalysis could be explained by a slightly deviating calibration bias.

The concordance between hemaPEN DBS concentrations (mean of three analyses) and whole blood concentrations (mean of two analyses) for both analytes is depicted by the Bland-Altman plots in Figure 3. For caffeine, an overall concentration difference of $-5.6 \%$ between whole blood and hemaPEN DBS was found, implying a slight underestimation of the hemaPEN DBS concentration compared to whole blood. For paraxanthine, a similar difference of $-4.4 \%$ was found. This concentration difference may be ascribed to a slight difference in calibration, since daily prepared calibration curves were used, and analyses were performed on different days. All mean hemaPEN DBS measurements differed less than $20 \%$ from the whole blood concentration for caffeine and for paraxanthine. The Bland-Altman comparison for whole liquid blood to $3 \mathrm{~mm}$ partial-punch DBS can be found in the supplementary data (Suppl. Data Figure S-1).

Furthermore, the concentration differences between $3 \mathrm{~mm}$ partial-punch DBS and whole blood and hemaPEN DBS vs. whole blood were plotted against the hct, as shown in Figure 4. In line with earlier observations, ${ }^{13,16}$ we found a pronounced hct bias when using subpunches of regular DBS (Figure 4A,D). When performing linear regression analysis to describe the \% concentration difference as a function of the hct, the slope of the regression line did not include zero for both caffeine and paraxanthine, indicating a significant hct effect. When evaluating what impact is imposed by the hct when comparing a sample with an hct of 0.20 vs. a sample with an hct of 0.50 , this implies concentration differences of 25.7 and $26.3 \%$, for caffeine and paraxanthine, respectively. Previously, we already demonstrated that our extraction procedure for conventional 


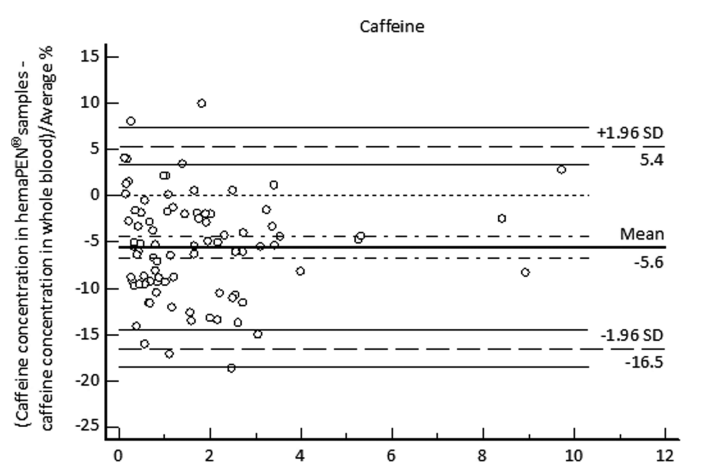

Mean of caffeine concentrations $(\mu \mathrm{g} / \mathrm{mL})$ in hemaPEN ${ }^{\ominus}$ samples and whole blood

Paraxanthine

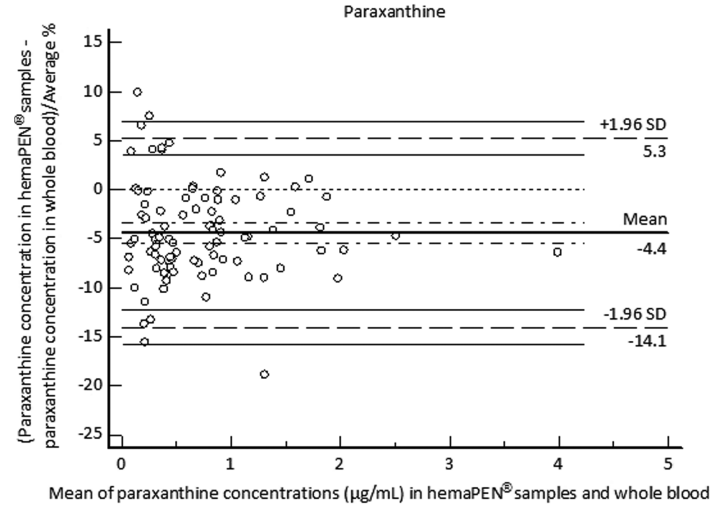

Figure 3. Bland-Altman plots for the comparison between whole blood and mean hemaPEN concentrations for caffeine $(n=88)$ and paraxanthine $(n=91)$. Mean differences and limits of agreement (LoA) are represented by full lines, and $95 \%$ confidence limits are represented by broken lines.

DBS was hct-independent, so this effect can be related to the spreading effect of the hct, rather than to an hct-imposed extractability issue. ${ }^{13,20}$ Yet, as we previously demonstrated that extraction at higher temperatures may overcome extractability issues, ${ }^{13,26}$ we repeated the analysis, using replicates of the partial-punch DBS that were extracted at 60 ${ }^{\circ} \mathrm{C}$ instead of at $22{ }^{\circ} \mathrm{C}$. Still, differences exceeding $25 \%$ were observed, thus excluding that hct-imposed extractability issues would play a role in this observation. When plotting the concentration differences between hemaPEN DBS versus whole blood, expressed as a \%, versus the hct, concentration differences of 10.2 and $5.7 \%$ were obtained when comparing a sample with an hct of 0.20 vs. 0.50 (Suppl. Data Figure S-2). Although this is much less than the effect observed for conventional DBS, we were surprised to find any impact of the hct. Statistical analysis demonstrated that the observed differences, though limited, were significant, as evidenced by the fact that the slope of the regression line was significantly different from zero. This unexpected finding was confirmed for both analytes by plotting in a similar manner the regression lines for the incurred hemaPEN DBS reanalyses: in both reanalyses and for both analytes, zero was not included in the 95\% confidence interval of the slope (Suppl. Data Figure S-2).

When considering samples with an hct of 0.20 vs. 0.50 , the $\%$ difference between the mean of the three hemaPEN DBS concentrations and those in whole blood was $6.90 \%$ for caffeine and $5.40 \%$ for paraxanthine (Figure 4B,E). From Figure 4, it is also clear that, although the impact of the hct reaches statistical significance, it is overall quite limited, certainly when comparing hemaPEN results with conventional partial-punch DBS results. Moreover, when considering the impact relative to an intermediate hct (0.40), the impact for the entire evaluated range $(0.17-0.53)$ remained within $6 \%$, which can be considered negligible.

As we considered it remarkable that, when using the hemaPEN device to generate DBS from blood with a lower hct, concentrations appeared to be slightly underestimated, we performed an additional experiment. This experiment, performed on an independent set of patient samples, involved full-spot analysis of DBS that were generated by pipetting 2.74 $\mu \mathrm{L}$ of patient blood samples with either low or high het values on prepunched $3 \mathrm{~mm}$ Whatman 903 filter paper discs (analyzed in triplicate) and comparison of the obtained results with those of the corresponding whole blood samples (analyzed in duplicate). For caffeine, 15 samples with an hct below 0.29 and 27 with an het above 0.45 were included. For paraxanthine, 15 samples with an het below 0.29 and 24 with
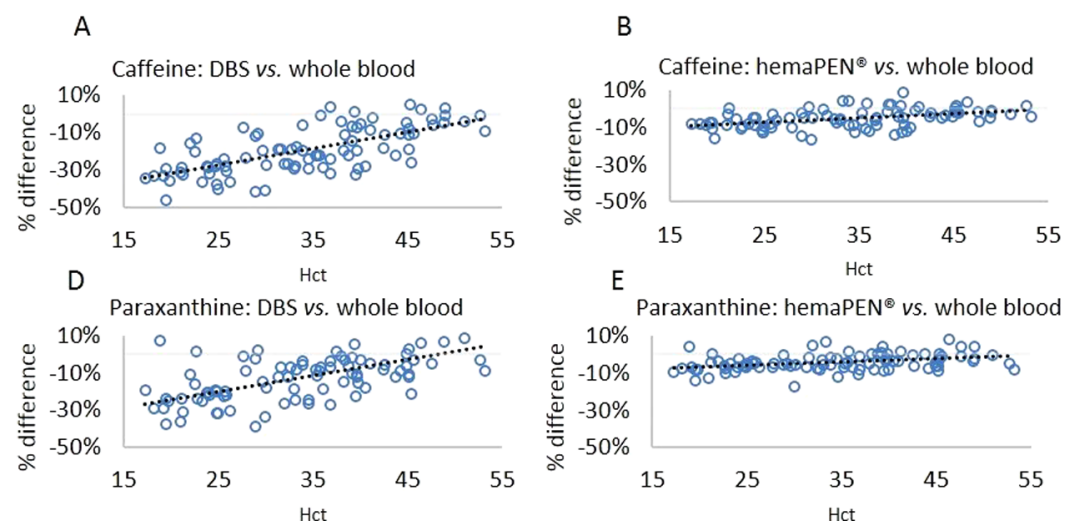

\section{C}
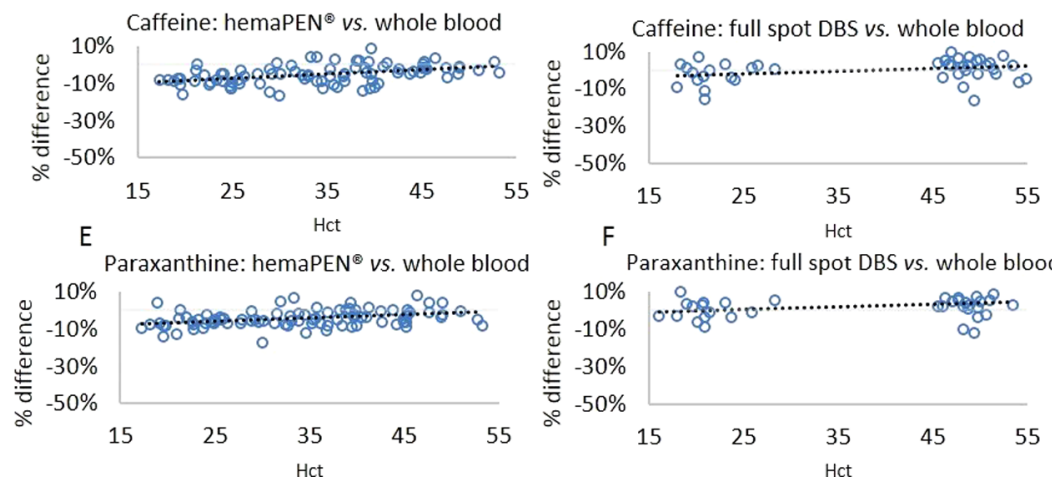

Paraxanthine: full spot DBS vs. whole blood

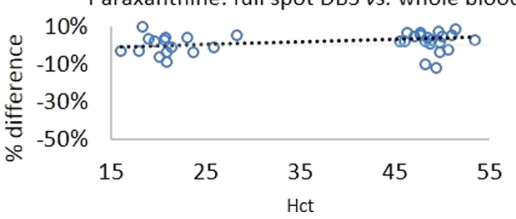

Figure 4. \% difference between $3 \mathrm{~mm}$ partial-punch DBS, hemaPEN DBS, or full $2.74 \mu \mathrm{L}$ DBS and whole blood concentrations, plotted against hct for caffeine (in panels A, B, and C respectively) and paraxanthine (in panels D, E, and F respectively). Broken lines represent linear regression lines. The respective slopes for $3 \mathrm{~mm}$ partial-punch DBS, hemaPEN DBS, and full-spot DBS were 0.0089 (95\%CI [0.006848; 00.010986]), 0.0023 (95\% CI $[0.00122 ; 0.00344])$, and $0.0013(95 \% \mathrm{CI}[-0.00012 ; 0.00268])$ for caffeine and $0.0086(95 \% \mathrm{CI}[0.00630 ; 0.01082])$, 0.0018 (95\%CI $[0.00075 ; 0.00275])$, and $0.0013(95 \% \mathrm{CI}[-0.000068 ; 0.002623])$ for paraxanthine. The respective intercepts for $3 \mathrm{~mm}$ partial-punch DBS, hemaPEN DBS, and full-spot DBS were -0.4995 (95\%CI $[-0.5728 ;-0.4262]),-0.1324(95 \% \mathrm{CI}[-0.1717 ;-0.0931])$, and $-0.0496(95 \% \mathrm{CI}$ $[-0.1072 ; 0.0081])$ for caffeine and $-0.4137(95 \% \mathrm{CI}[-0.4938 ;-0.3335]),-0.1018(95 \% \mathrm{CI}[-0.1373 ;-0.0663])$, and $-0.0277(95 \% \mathrm{CI}$ $[-0.0824 ; 0.0269])$ for paraxanthine. 
an hct above 0.45 were included. One data point was excluded, because the $\mathrm{CV}$ of the triplicate analysis of a full DBS exceeded $15 \%$ for both analytes, indicating a technical issue. Based on a generalized ESD test, one full-spot DBS sample was excluded because of a deviating paraxanthine to caffeine ratio. The $\%$ difference was calculated as (mean DBS concentration - mean whole blood concentration)/mean whole blood concentration and was plotted against the hct. Linear regression analysis did not reveal a significant trend as a function of the hct for both analytes, as zero was included in the $95 \% \mathrm{CI}$ of both slopes, as depicted in Figure 4C,F. Re-evaluation of the hemaPENwhole blood comparison, only taking into account the samples with hct values below 0.29 and above 0.45 still yielded a significant trend. Based on the results of the additional experiment including full-spot analysis of volumetrically applied DBS, it cannot be stated that the small hct bias observed for the hemaPEN devices is related to the analytical procedure. As in the case of blood with low hct, the capillaries are not only filled but also emptied faster, ${ }^{19,27}$ we hypothesize that this quick emptying might result in somewhat more retention of liquid in the capillaries.

In a second set of experiments, we evaluated the robustness of the hemaPEN to the effects of blood sample volume, device lot, analytical operator, and storage stability. A subset of 25 patient samples, with an hct ranging from 0.17 to 0.57 , was used for this purpose. When presenting $50 \mu \mathrm{L}$ instead of $20 \mu \mathrm{L}$ blood drops to the hemaPEN, 92 and $87 \%$ of the caffeine and paraxanthine concentrations differed less than $20 \%$, thereby complying with the preset criterion of $67 \%$. When using another lot of hemaPENs, all results lay within $20 \%$ of those obtained using the first hemaPEN lot. Respectively, 8 and $20 \%$ of the caffeine and paraxanthine samples sampled by another operator showed a \% difference with the first operator above $20 \%$. Hence, also here the preset acceptance criterion was met. In supplementary data (Suppl. Data Figure S-3), the \% differences for all these subsets are depicted. Mean differences compared to the original were 4,2 , and $8 \%$ for the $50 \mu \mathrm{L}$, another batch, and other operator sets respectively, for caffeine and 8,6 , and $13 \%$ for paraxanthine. To also evaluate sample stability in and extractability from authentic patient samples, rather than solely in spiked QCs, the stability of caffeine and paraxanthine in hemaPEN devices, used to generate DBS from the aforementioned 25 patient samples, was assessed after storage for 4 days at $60{ }^{\circ} \mathrm{C}$. When compared to the original hemaPEN concentrations, a mean deviation of $0.3 \%$ for caffeine and of $8 \%$ for paraxanthine was found, meeting the preset acceptance criterion of $\pm 15 \%$ and confirming that there is neither a stability nor an extractability issue (Suppl. Data Figure S-3).

Samples from 10 volunteers who confirmed not to be caffeine abstinent were included in a transport simulation study. All volunteers received a brief description about usage of the device; $3 / 10$ had used the device before. For $6 / 10$ volunteers, duplicate samples for both hemaPENs could be obtained. For two volunteers, the hemaPEN that stayed in the laboratory contained only one DBS. For the remaining two volunteers, sampling with one pen failed, and as a consequence, no comparison could be made. Hence, data from eight different volunteers were included in data analysis. When a duplicate sample was available in the pen that stayed in the lab, the mean was calculated and considered as the reference concentration. In the two cases where only a single sample was available, this was considered the reference.
For each volunteer, the \% difference between the hemaPEN DBS concentration of the pen that stayed in the lab and that of the hemaPEN DBS after transport is depicted in Figure 5. In

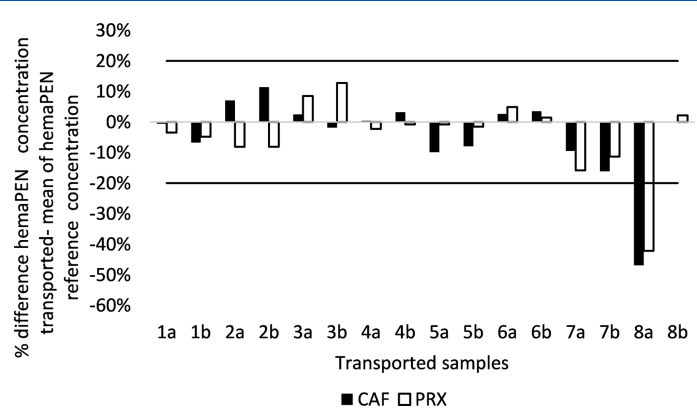

Figure 5. Deviation (\%) between the caffeine and paraxanthine concentrations determined after transport vs. the concentrations determined in the hemaPEN DBS that stayed in the lab, which are considered as the reference.

all but one case (sample 8a), the deviation was less than $20 \%$. We have no clear-cut explanation for the deviating result that suggests overfilling of a DBS, especially as in a replicate from the same hemaPEN (sample $8 \mathrm{~b}$ ) for both analytes, the difference from the reference concentrations was less than $2.5 \%$. The median absolute deviation was $5 \%$ for both analytes.

Last, in addition to the analytical performance, we also assessed the success rate (technical performance) of the hemaPENs that had been used for the validation (calibrators and QCs) and for the patient sample study. Both the filling and the emptying of all four capillaries were scored. In Figure 6, the

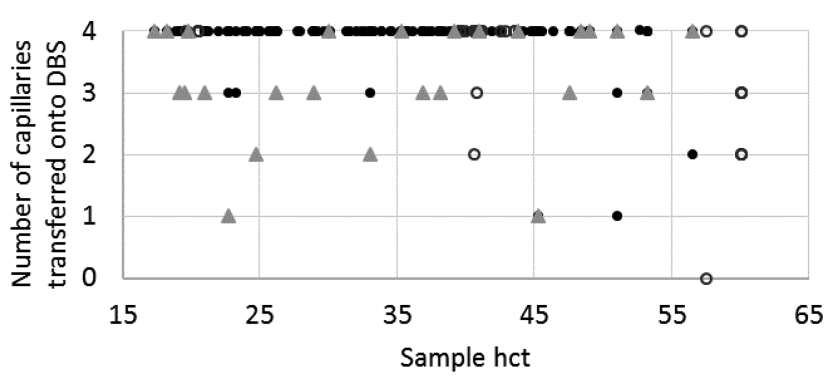

- Patient samples $20 \mu \mathrm{L}(\mathrm{n}=176)$ ○ Validation samples $(\mathrm{n}=93)$

$\triangle$ Patient samples $50 \mu \mathrm{L}(\mathrm{n}=25)$

Figure 6. hemaPEN functionality expressed as the number of capillaries transferred onto the DBS prepunch in function of the hct of the venous blood sample.

number of capillaries transferred is plotted as a function of the hct (filling had for all the venous samples a $100 \%$ success rate). For $20 \mu \mathrm{L}$ blood samples with an hct above 0.50 , we observed an increased failure rate in emptying of the capillaries: for 12 / 23 samples with an hct above 0.50 , less than 4 spots were generated from completely filled capillaries, which contrasts with only 6 out of 246 samples with an hct below 0.50 for which less than 4 spots were obtained. This might be relevant for pediatric populations, since for this population, the use of microsampling is a substantial advantage, and hct values are generally higher, values above 0.50 not being exceptional. As in this study the share of samples with a high hct (above 0.50) was limited $(n=23)$, further research is warranted to determine to what extent this poses a true problem. 
Also, for the set of 25 samples where $50 \mu \mathrm{L}$ of blood was presented to the hemaPEN (Figure 6, triangles), transfer onto the prepunched disc seemed to be less efficient (in 13 cases, no successful generation of four DBS), although also here the sample size was limited. This might be due to an excess of blood clogging the capillary inlet, thereby inhibiting capillary action to take place. Following clicking the hemaPEN in its base, capillary action is the driving force for the transfer of the filled capillaries onto the prepunched filter paper discs inside the hemaPEN. Obviously, in a home sampling scenario, blood will not be pipetted into an Eppendorf to sample blood with a hemaPEN device but will be obtained from a fingerstick. In this scenario, clogging of the capillaries could also take place when a blood drop is generated that is too large. The functionality when dealing with patients having a high hct and in cases where a large blood drop is present should further be investigated-however, this was not among the aims of our study. Anyway, to guarantee the successful generation of DBS using the hemaPEN, we feel that training of patients or caregivers involved with this sampling technique will be an essential element. On the other hand, the hemaPEN automatically generates four replicate spots in one pen. In this study, in only a few cases, none (one case) or only one (four cases) out of the four samples could be generated. Therefore, sample analysis will in the far majority of the cases still be possible, even with high-hct samples or when large volumes of blood are in contact with the capillary inlet.

\section{CONCLUSION}

An LC-MS/MS method for the determination of caffeine and its metabolite paraxanthine in DBS generated using hemaPEN devices was successfully validated based on the European Medicines Agency (EMA) and U.S. Food and Drug Administration (FDA) guidelines on bioanalytical method validation. ${ }^{21,22}$ Since all preset acceptance criteria were met, it can be concluded that these devices allow the accurate and precise collection of a fixed amount $(2.74 \mu \mathrm{L})$ of blood. The hemaPEN device was proposed as an alternative, volumetric, hct-independent DBS device. ${ }^{19}$ The potential of these devices to cope with the hct-based bias, seen in regular subpunched DBS, was evaluated by analyzing 88 and 91 authentic patient samples (hct range 0.17 to 0.53 ) for caffeine and paraxanthine. The comparison of analyte concentrations measured in hemaPEN DBS with those in liquid venous whole blood samples revealed a slight (but statistically significant) hct-based bias (6.90 and 5.40\% concentration differences for caffeine and paraxanthine, respectively, over a $0.20-0.50$ hct range). This effect, which we could not attribute to the analytical procedure, is very limited when compared to subpunched DBS, showing concentration differences with whole blood of $\geq 25 \%$ over this hct range. Moreover, when considering the impact relative to an intermediate hct (0.40), the impact remained within $6 \%$ for the entire evaluated hct range $(0.17-0.53)$ and may be considered negligible. Evaluation of the hemaPEN robustness to the effects of blood sample volume, device lot, analytical operator, and storage stability passed the preset criteria for all of the above-mentioned variables. Furthermore, transport of eight capillary samples from eight healthy volunteers by postal services revealed no instability of both analytes. The technical performance of the hemaPEN when dealing with patients having a high het and in cases where a large blood drop is present should be further investigated. Based on all the above findings, the hemaPEN device shows good potential as an alternative for conventional DBS, being an almost hctindependent microsampling device, allowing volumetric collection of DBS. Further evaluation using true capillary blood samples obtained by fingerstick is warranted to further evaluate the performance of the device under realistic circumstances.

\section{ASSOCIATED CONTENT}

\section{S Supporting Information}

The Supporting Information is available free of charge on the ACS Publications website at DOI: 10.1021/acs.analchem.9b03179.

Table S-1: Analyte and IS-compensated matrix effect; Table S-2: Stability study; Figure S-1: Bland-Altman comparison for whole liquid blood to $3 \mathrm{~mm}$ partialpunch DBS; Figure S-2: Regression analyses of the individual hemaPEN-whole blood differences as a function of hct; Figure S-3: Box-and-whisker plot evaluation of hemaPEN robustness to sample volume, device lot, analytical operator, and sample stability (PDF)

\section{AUTHOR INFORMATION}

\section{Corresponding Author}

*E-mail: christophe.stove@ugent.be; Tel.: +32 92648135 .

ORCID

Lucía Paniagua-González: 0000-0002-2278-717X

Christophe P. Stove: 0000-0001-7126-348X

\section{Author Contributions}

The manuscript was written through contributions of all authors. All authors have approved the final version of the manuscript.

\section{Notes}

The authors declare no competing financial interest.

\section{ACKNOWLEDGMENTS}

The authors acknowledge Prof. Dr. Veronique Stove and the team of the core lab from Ghent University Hospital. Also acknowledged are Annelies Cannaert, Lisa Delahaye, and Jana Verstraete, for their assistance with patient sample collection, as well as the volunteers participating in this study. S.D. would like to thank the Research Foundation-Flanders (FWO) for granting her a $\mathrm{PhD}$ fellowship (application number: $11 \mathrm{~F} 3119 \mathrm{~N})$. L.P.-G. would like to thank the Conselleria de Cultura, Educación e Ordenación Universitaria, Xunta de Galicia, for her predoctoral (ED481A-2018/059) contract. The team from Trajan (especially Pawanbir Singh and Florian Lapierre) and Dr. Neil Spooner are acknowledged for helpful discussions.

\section{REFERENCES}

(1) Edelbroek, P. M.; van der Heijden, J.; Stolk, L. M. Ther. Drug Monit. 2009, 31, 327-336.

(2) Stove, C. P.; Ingels, A. S.; De Kesel, P. M.; Lambert, W. E. Crit. Rev. Toxicol. 2012, 42, 230-243.

(3) Capiau, S.; Alffenaar, J.-W.; Stove, C. P. In Clinical Challenges in Therapeutic Drug Monitoring 2016, 279-336.

(4) Wilhelm, A. J.; den Burger, J. C.; Swart, E. L. Clin. Pharmacokinet. 2014, 53, 961-973.

(5) Sadones, N.; Capiau, S.; De Kesel, P. M.; Lambert, W. E.; Stove, C. P. Bioanalysis 2014, 6, 2211-2227.

(6) Cox, H.; Eichner, D. Anal. Chem. 2017, 89, 10029-10036. 
(7) De Kesel, P. M. M.; Lambert, W. E.; Stove, C. P. Clin. Pharmacokinet. 2014, 53, 763-771.

(8) De Kesel, P. M.; Sadones, N.; Capiau, S.; Lambert, W. E.; Stove, C. P. Bioanalysis 2013, 5, 2023-2041.

(9) Abu-Rabie, P.; Denniff, P.; Spooner, N.; Chowdhry, B. Z.; Pullen, F. S. Anal. Chem. 2015, 87, 4996-5003.

(10) Velghe, S.; Delahaye, L.; Stove, C. P. J. Pharm. Biomed. Anal. 2019, 163, 188-196.

(11) Xie, I.; Xu, Y.; Anderson, M.; Wang, M.; Xue, L.; Breidinger, S.; Goykhman, D.; Woolf, E. J.; Bateman, K. P. J. Pharm. Biomed. Anal. 2018, 156, 58-66.

(12) Denniff, P.; Spooner, N. Anal. Chem. 2014, 86, 8489-8495.

(13) De Kesel, P. M.; Lambert, W. E.; Stove, C. P. Anal. Chim. Acta 2015, 881, 65-73.

(14) Leuthold, L. A.; Heudi, O.; Déglon, J.; Raccuglia, M.; Augsburger, M.; Picard, F.; Kretz, O.; Thomas, A. Anal. Chem. 2015, 87, 2068-2071.

(15) Nakahara, T.; Otani, N.; Ueno, T.; Hashimoto, K. J. Chromatogr. B: Anal. Technol. Biomed. Life Sci. 2018, 1087-1088, 70-79.

(16) Velghe, S.; Stove, C. P. Anal. Chem. 2018, 90, 12893-12899.

(17) Lenk, G.; Ullah, S.; Stemme, G.; Beck, O.; Roxhed, N. Anal. Chem. 2019, 91, 5558-5565.

(18) Capiau, S.; Veenhof, H.; Koster, R.; Bergqvist, Y.; Boettcher, M.; Halmingh, O.; Keevil, B.; Koch, B.; Linden, R.; Pistos, C.; Stolk, L.; Touw, D.; Stove, C.; Alffenaar, J. W. Ther. Drug Monit. 2019, 41, 409.

(19) Neto, R.; Gooley, A.; Breadmore, M. C.; Hilder, E. F.; Lapierre, F. Anal. Bioanal. Chem. 2018, 410, 3315-3323.

(20) De Kesel, P. M.; Lambert, W. E.; Stove, C. P. Bioanalysis 2014, 6, 3011-3024.

(21) European Medicines Agency. Guideline on bioanalytical method validation, 2012.

(22) U.S. Department of Health and Human Services; Food and Drug Administration; Center for Drug Evaluation and Research (CDER); Center for Veterinary Medicine (CVM). Bioanalytical Method Validation Guidance on Industry, 2018.

(23) Desharnais, B.; Camirand-Lemyre, F.; Mireault, P.; Skinner, C. D. J. Anal. Toxicol. 2017, 41, 261-268.

(24) Wille, S. M. R.; Peters, F. T.; Di Fazio, V.; Samyn, N. Accredit. Qual. Assur. 2011, 16, 279-292.

(25) Matuszewski, B. K. J. J. Chromatogr. B: Anal. Technol. Biomed. Life Sci. 2006, 830, 293-300.

(26) Velghe, S.; Stove, C. P. Anal. Bioanal. Chem. 2018, 410, 23312341.

(27) Lapierre, F.; Gooley, A.; Breadmore, M. Langmuir 2017, 33, $14220-14225$. 